\title{
Computational Comparison of Continuous and Discontinuous Galerkin Time-Stepping Methods for Nonlinear Initial Value Problems
}

\author{
Bärbel Janssen and Thomas P. Wihler
}

\begin{abstract}
This article centers on the computational performance of the continuous and discontinuous Galerkin time stepping schemes for general first-order initial value problems in $\mathbb{R}^{n}$, with continuous nonlinearities. We briefly review a recent existence result for discrete solutions from [6], and provide a numerical comparison of the two time discretization methods.
\end{abstract}

Key words: Galerkin time discretizations, numerical approximation of initial value problems, higher order time stepping methods.

\section{Introduction}

In this paper we focus on (possibly high-order) continuous and discontinuous Galerkin (cG and dG, respectively) time stepping discretizations as applied to initial value problems of the form

$$
\begin{aligned}
& u^{\prime}(t)=\mathscr{F}(t, u(t)), \quad t \in(0, T), \\
& u(0)=u_{0} .
\end{aligned}
$$

Here, $u:(0, T) \rightarrow \mathbb{R}^{n}$, for some $n \in \mathbb{N}$ and $T>0$, is an unknown solution. The initial vector $u_{0} \in \mathbb{R}^{n}$ prescribes the solution $u$ at the start-up time $t=0$, and $\mathscr{F}$ : $[0, T] \times \mathbb{R}^{n} \rightarrow \mathbb{R}^{n}$ is a possibly nonlinear, continuous operator. We will usually omit to explicitly write the dependence on the first argument $t$.

Bärbel Janssen

Department of High Performance Computing and Visualization, School of Computer Science and Communication, KTH Royal Institute of Technology, Stockholm, Sweden e-mail: barbel@kth.se

Thomas P. Wihler

Mathematics Institute, University of Bern, Switzerland e-mail: wihler@math.unibe.ch 
Galerkin-type time stepping methods for initial-value problems are based on weak formulations. For both the $\mathrm{cG}$ and the $\mathrm{dG}$ schemes, the test spaces constitute of polynomials that are discontinuous at the time nodes. In this way, the discrete Galerkin formulations decouple into local problems on each time step, and the discretizations can hence be understood as implicit one-step schemes. Galerkin time stepping methods have been analyzed for ordinary differential equations (ODEs), e.g., in $[2,3,4,5,7,10,13]$.

In the current article, we will start by reviewing the definitions of the cG and $\mathrm{dG}$ schemes of arbitrary order in Section 2. Furthermore, we will recall the recent work [6] which shows that the existence of discrete $\mathrm{cG}$ and $\mathrm{dG}$ solutions for continuous nonlinearities is independent of the approximation order and only requires the local time steps to be sufficiently small (and thereby generalizes the previous works [10, 13], where Lipschitz continuous nonlinearities where considered). The focus of this work is to provide a computational comparison of the two schemes in Section 4.

Throughout the paper, we shall use the following notation: For an interval $I=$ $(a, b), a<b$, the space $C^{0}(\bar{I})$ consists of all functions $u: \bar{I} \rightarrow \mathbb{R}^{n}$ that are continuous on $\bar{I}$. Moreover, introducing, for $1 \leq p<\infty$, the norm

$$
\|u\|_{L^{p}(I)}=\left(\int_{I}|u(t)|^{p} \mathrm{~d} t\right)^{1 / p}
$$

and, for $p=\infty$, the norm $\|u\|_{L^{\infty}(I)}=\operatorname{ess} \sup _{t \in I}|u(t)|$, we write $L^{p}(I)$ to signify the space of measurable functions $u: I \rightarrow \mathbb{R}^{n}$ so that the corresponding norm is bounded. We note that $L^{2}(I)$ is a Hilbert space with the inner product

$$
(u, v)_{L^{2}(I)}=\int_{I}(u(t), v(t)) \mathrm{d} t .
$$

Here, $(\cdot, \cdot)$ and $|\cdot|$ denote the standard dot product and Euclidean norm in $\mathbb{R}^{n}$, respectively.

\section{Galerkin Time Stepping}

On an interval $I=[0, T]$, consider time nodes $0=t_{0}<t_{1}<\cdots<t_{M-1}<t_{M}=T$ which introduce a time partition $\mathscr{M}=\left\{I_{m}\right\}_{m=1}^{M}$ of $I$ into $M$ open time intervals $I_{m}=$ $\left(t_{m-1}, t_{m}\right), m=1, \ldots, M$. The length $k_{m}=t_{m}-t_{m-1}$ of a time interval (which may vary locally) is called the $m^{\text {th }}$ time step. Furthermore, we let $r \geq 0$ to be a (global) polynomial degree, which takes the role of an approximation order. Then, given $s \in$ $\mathbb{N}_{0}$, the set

$$
\mathscr{P}^{s}(J)=\left\{p \in C^{0}(\bar{J}): p(t)=\sum_{i=0}^{s} x_{i} t^{i}, x_{i} \in \mathbb{R}^{n}\right\}
$$


signifies the space of all polynomials of degree at most $s$ on an interval $J \subset \mathbb{R}$ with values in $\mathbb{R}^{n}$.

The $\mathrm{cG}(r)$ and $\mathrm{dG}(r)$ time marching methods on $\mathscr{M}$ will seek solutions that locally belong to the spaces $\mathscr{P}^{r+1}\left(I_{m}\right)$ and $\mathscr{P}^{r}\left(I_{m}\right)$, respectively. We emphasize that, for both schemes, the local test space is $\mathscr{P}^{r}\left(I_{m}\right)$.

\subsection{The cG Method}

With the notation above, the $\mathrm{cG}(r)$ time marching scheme is iteratively given as follows: For a prescribed initial vector $U_{m-1}:=\left.U\right|_{I_{m-1}}\left(t_{m-1}\right) \in \mathbb{R}^{n}$ (with $U_{0}:=u_{0}$, where $u_{0} \in \mathbb{R}^{n}$ is the initial vector from (2)), we find $\left.U\right|_{I_{m}} \in \mathscr{P}^{r+1}\left(I_{m}\right)$ through the weak formulation

$$
\begin{aligned}
\int_{I_{m}}\left(U^{\prime}, V\right) \mathrm{d} t & =\int_{I_{m}}(\mathscr{F}(U), V) \mathrm{d} t \quad \forall V \in \mathscr{P}^{r}\left(I_{m}\right), \\
U\left(t_{m-1}\right) & =U_{m-1},
\end{aligned}
$$

for any $1 \leq m \leq M$. Notice that, in order to enforce the initial condition on each individual time step (and thereby to obtain a globally continuous solution $U$ on $(0, T)$ ), the local trial space possesses one degree of freedom more than the local test space.

Introducing the (local) $L^{2}$-projection $\Pi_{m}^{r}: L^{2}\left(I_{m}\right) \rightarrow \mathscr{P}^{r}\left(I_{m}\right)$ onto $\mathscr{P}^{r}\left(I_{m}\right)$ given by

$$
\int_{I_{m}}\left(v-\Pi_{m}^{r} v, w\right) \mathrm{d} t=0 \quad \forall w \in \mathscr{P}^{r}\left(I_{m}\right),
$$

the following result is quite elementary to deduce:

Proposition 1. A function $U \in \mathscr{P}^{r+1}\left(I_{m}\right)$ is a solution of (3) if and only if $U$ satisfies the fixed point equation

$$
U(t)=U_{m-1}+\int_{t_{m-1}}^{t} \Pi_{m}^{r} \mathscr{F}(U) d \tau
$$

for any $t \in I_{m}$.

\subsection{The dG Method}

In order to define the discontinuous Galerkin scheme, some additional notation is required: We define the one-sided limits of a piecewise continuous function $U$ at each time node $t_{m}$ by

$$
U_{m}^{+}:=\lim _{s \searrow 0} U\left(t_{m}+s\right), \quad U_{m}^{-}:=\lim _{s \nearrow 0} U\left(t_{m}+s\right) .
$$


Then, the discontinuity jump of $U$ at $t_{m}$, for $0 \leq m \leq M-1$, is defined by $[[U]]_{m}=$ $U_{m}^{+}-U_{m}^{-}$; for $m=0$ we set $U_{0}^{-}=u_{0}$, where $u_{0}$ is the initial vector from (2).

With these definitions the $\mathrm{dG}(r)$ time stepping method for (1)-(2) reads: Find $\left.U\right|_{I_{m}} \in \mathscr{P}^{r}\left(I_{m}\right)$ such that

$$
\int_{I_{m}}\left(U^{\prime}, V\right) \mathrm{d} t+\left([[U]]_{m-1}, V_{m-1}^{+}\right)=\int_{I_{m}}(\mathscr{F}(U), V) \mathrm{d} t \quad \forall V \in \mathscr{P}^{r}\left(I_{m}\right),
$$

for any $1 \leq m \leq M$. We underline that, in contrast to the continuous Galerkin formulation, the local trial and test spaces are the same for the discontinuous Galerkin scheme. This is due to the fact that the initial values are weakly imposed (by means of an upwind flux) on each time interval.

In order to derive a fixed-point formulation for the dG scheme as in (4), we revisit [11, Section 4.1] to define a lifting operator, for $1 \leq m \leq M$,

$$
\mathrm{L}_{m}^{r}: \mathbb{R}^{n} \rightarrow \mathscr{P}^{r}\left(I_{m}\right)
$$

by

$$
\int_{I_{m}}\left(\mathrm{~L}_{m}^{r}(z), V\right) \mathrm{d} t=\left(z, V_{m-1}^{+}\right) \quad \forall V \in \mathscr{P}^{r}\left(I_{m}\right), z \in \mathbb{R}^{n} .
$$

Then, looking at the discrete derivative operator

$$
\chi: \mathscr{P}^{r}\left(I_{m}\right) \rightarrow \mathscr{P}^{r}\left(I_{m}\right), \quad U \mapsto \chi(U)=U^{\prime}+\mathrm{L}_{m}^{r}\left(U_{m-1}^{+}\right),
$$

we recall the following result from [6].

Proposition 2. The operator $\chi$ from (6) is an isomorphism, and satisfies the bound, for any $p \in[1, \infty]$,

$$
\left\|\chi^{-1}(U)\right\|_{L^{\infty}\left(I_{m}\right)} \leq 2 k_{m}^{1-1 / p}\|U\|_{L^{p}\left(I_{m}\right)} \quad \forall U \in \mathscr{P}^{r}\left(I_{m}\right)
$$

Moreover, a function $U \in \mathscr{P}^{r}\left(I_{m}\right)$ is a solution of (5) if and only if the fixed point equation

$$
U=U_{m-1}^{-}+\chi^{-1}\left(\Pi_{m}^{r} \mathscr{F}(U)\right)
$$

is fulfilled.

Remark 1 . We note that the discrete operator $\chi$ from (6) is closely related to the (parabolic) reconstruction operator as discussed in, e.g., [8].

\section{Existence of Discrete Galerkin Solutions}

The well-known Peano Theorem (see, e.g., [12]) guarantees the existence of $C^{1}$ solutions $u$ of (1)-(2) within some limited time range, $t \in\left(0, T^{\star}\right)$, for some $T^{\star}>0$. Notice that the existence interval for solutions may be arbitrarily small even for 
smooth $\mathscr{F}$ : For instance, the initial value problem (1)-(2) may exhibit solutions that may become unbounded in finite time; to give an example, let us consider the initial value problem of finding a $\mathbb{R}$-valued function $u$ which satisfies

$$
u^{\prime}(t)=|u(t)|^{\beta-1} u(t), \quad u(0)=1
$$

for a given constant $\beta>1$. It is elementary to check that

$$
u(t)=(1-(\beta-1) t)^{\frac{1}{1-\beta}}
$$

is a solution of (8), and we see that there appears a blow-up as $t \nearrow T^{\star}:=\frac{1}{\beta-1}$.

Based on the fixed point equations (4) and (7) for the $\mathrm{cG}$ and $\mathrm{dG}$ schemes, respectively, it is possible to prove the ensuing existence result for solutions of (5), see [6]:

Theorem 1. Let $1 \leq m \leq M$, and suppose that, for some $\kappa_{m}>0$,

$$
K_{m}^{\kappa_{m}}:=\sup _{(t, y) \in I_{m} \times B_{\kappa_{m}}}|\mathscr{F}(t, y)|<\infty,
$$

where $B_{\kappa_{m}}=\left\{y \in \mathbb{R}^{n}:\left|y-U_{m-1}^{-}\right| \leq \kappa_{m}\right\}$. Then, if the local time step is chosen such that

$$
k_{m} \leq \frac{\kappa_{m}}{C_{\mathrm{ex}} K_{m}^{K_{m}}},
$$

where

$$
C_{\mathrm{ex}}=\left\{\begin{array}{l}
1 \text { for the } c G(r) \text { scheme } \\
2 \text { for the } d G(r) \text { scheme }
\end{array}\right.
$$

then the $c G(r)$ and $d G(r)$ methods from (3) and (5), respectively, on the time interval $I_{m}$ each possess at least one solution in $M_{m}^{\kappa_{m}}:=\left\{Y \in \mathscr{P}^{r+2-C_{\mathrm{ex}}}\left(I_{m}\right): Y(t) \in\right.$ $\left.B_{\kappa_{m}} \forall t \in \bar{I}_{m}\right\}$. In particular, the existence of discrete Galerkin solutions is independent of the polynomial degree $r$.

Remark 2. We note that Theorem 1 still holds true for varying polynomial degrees on each time interval.

\section{Numerical Experiments}

We will now compare the $\mathrm{cG}$ and $\mathrm{dG}$ discretizations by means of a few numerical tests. Specifically, we consider the initial value problem (8) for the linear case $\beta=1$,

$$
u^{\prime}(t)=u(t), \quad t \geq 0, \quad u(0)=1
$$

as well as for the nonlinear case $\beta=2$,

$$
u^{\prime}(t)=u(t)^{2}, \quad t \geq 0, \quad u(0)=1
$$


The former problem has an analytic exact solution which is given by $u(t)=\exp (t)$. For $\beta=2$, the exact solution is $u(t)=(1-t)^{-1}$, and features a blow-up as $t \nearrow 1$.

The time meshes in our computations are based on the existence criterion from Theorem 1, i.e., the individual time steps are chosen according to (9) (independently of the polynomial degree $r$ ). For $\beta=1$ and some $\kappa_{m}>0$ there holds that

$$
K_{m}^{\kappa_{m}}=\sup _{\left|y-U_{m-1}^{-}\right| \leq \kappa_{m}}|y|=\kappa_{m}+\left|U_{m-1}^{-}\right| .
$$

Hence, for $k_{m}$ in (9) we obtain

$$
k_{m} \leq \frac{\kappa_{m}}{C_{\mathrm{ex}}\left(\kappa_{m}+\left|U_{m-1}^{-}\right|\right)} \rightarrow C_{e x}^{-1}
$$

as $\kappa_{m} \rightarrow \infty$, where $C_{\mathrm{ex}}$ is the constant from (10). In our experiments we shall choose

$$
k_{m}=\frac{1}{2 C_{\mathrm{ex}}} \quad(\beta=1) .
$$

For $\beta=2$, it has been shown in [6] that the maximal possible time step according to $(9)$ is given by

$$
k_{m}=\frac{1}{4 C_{\mathrm{ex}}\left|U_{m-1}^{-}\right|} \quad(\beta=2) .
$$

Here, $C_{\mathrm{ex}}$ is again the constant from (10). Incidentally, while the time steps for $\beta=1$ are chosen to be of constant size, the time mesh for $\beta=2$ turns out to be geometrically refined towards the blow-up point at $T=1$.

In order to deal with the nonlinearities, the Newton method will be applied. We note that, for $\beta=2$ close to the blow-up, the Newton iterations may deteriorate or take a long time to converge. If the Newton method fails to converge, we simply stop the time iteration.

In Figures 1-4 we compare the performance of the $\mathrm{dG}(r)$ and the $\mathrm{cG}(r+1)$ time stepping methods as applied to our model problem (8); note that, for given $r \geq 0$, these methods feature the same number of degrees of freedom on each time step (as they are both based on the same test spaces). In each of the figures below we display the ratio of the $\mathrm{cG}(r+1)$ and $\mathrm{dG}(r)$ errors for different error types, including the accumulated $L^{2}$ errors, the $L^{\infty}$ errors, and the nodal end time errors, for different problem parameters. More precisely, we use the following notation:

- Accumulated $L^{2}$ error:

$$
\frac{\left\|u-U^{\mathrm{cG}}\right\|_{L^{2}\left(0, t_{m}\right)}}{\left\|u-U^{\mathrm{dG}}\right\|_{L^{2}\left(0, t_{m}\right)}}, \quad m \geq 1 ;
$$

- $L^{\infty}$ error:

$$
\frac{\left\|u-U^{\mathrm{cG}}\right\|_{L^{\infty}\left(0, t_{m}\right)}}{\left\|u-U^{\mathrm{dG}}\right\|_{L^{\infty}\left(0, t_{m}\right)}}, \quad m \geq 1
$$


- Nodal end time error:

$$
\frac{\left|e_{m}^{c \mathrm{G}}\right|}{\left|e_{m}^{\mathrm{dG}}\right|}=\frac{\left|u\left(t_{m}\right)-U_{m}^{\mathrm{cG}}\right|}{\left|u\left(t_{m}\right)-\left(U^{\mathrm{dG}}\right)_{m}^{-l}\right|}, \quad m \geq 1
$$

Here, $u$ is the exact solution of (8) (for $\beta \in\{1,2\}$ ), and $U^{\mathrm{cG}}$ and $U^{\mathrm{dG}}$ denote the corresponding $\mathrm{cG}(r+1)$ and $\mathrm{dG}(r)$ solutions defined by (3) and (5), respectively. In our experiments we perform tests for both $C_{\mathrm{ex}}=1$ (existence for the $\mathrm{cG}$ method) and $C_{\mathrm{ex}}=2$ (existence for the $\mathrm{dG}$ method) for both schemes; cf. (10).

\section{Discussion of the results}

In terms of the $L^{2}$ and $L^{\infty}$ errors in the low-order context, the cG method seems to perform better than the dG scheme in both the smooth $(\beta=1)$ as well as in the blow-up $(\beta=2)$ case. For $\beta=1$, however, the ratios tend to a limit just below 1 for increasing polynomial degrees $r$. This behavior is similar for $\beta=2$ (away from the blow-up time $T=1$ ), although here we observe that the ratios seem to stabilize slightly above 1 for higher $r$.

For the ratios of the nodal end time errors, we only show results for polynomial degrees $r=0,1,2,3$, and for $\beta=1$; indeed, for higher polynomial degrees (and $\beta=2$ away from the blow-up) the nodal end time errors become quickly close to machine precision due to well-known super convergence effects at nodes in Galerkin time stepping discretizations. We observe that the $\mathrm{cG}$ method performs again better than the $\mathrm{dG}$ method; for increasing polynomial degree, the dominance of the $\mathrm{cG}$ scheme over the $\mathrm{dG}$ scheme becomes even more pronounced. This behavior is not surprising since the super convergence regime of the $\mathrm{cG}$ scheme for smooth solutions is (at least theoretically) superior to the $\mathrm{dG}$ method (see the papers [1,9] for related super convergence results for Galerkin methods).

In conclusion, both discretization schemes perform similarly in the high-order context, whereas the $\mathrm{cG}$ method seems a little more favorable in the low-order setting for the examples considered here.

Acknowledgements TW would like to thank the scientific committee and the local organizers of ICOSAHOM 2014 for the conference invitation to Salt Lake City. Furthermore, he acknowledges the financial support of the Swiss National Science Foundation.

\section{References}

1. Adjerid, S., Devine, K.D., Flaherty, J.E., Krivodonova, L.: A posteriori error estimation for discontinuous Galerkin solutions of hyperbolic problems. Comput. Methods Appl. Mech. Engrg. 191(11-12), 1097-1112 (2002)

2. Bangerth, W., Rannacher, R.: Adaptive Finite Element Methods for Differential Equations. Lectures in Mathematics, ETH Zürich. Birkhäuser Verlag, Basel (2003) 

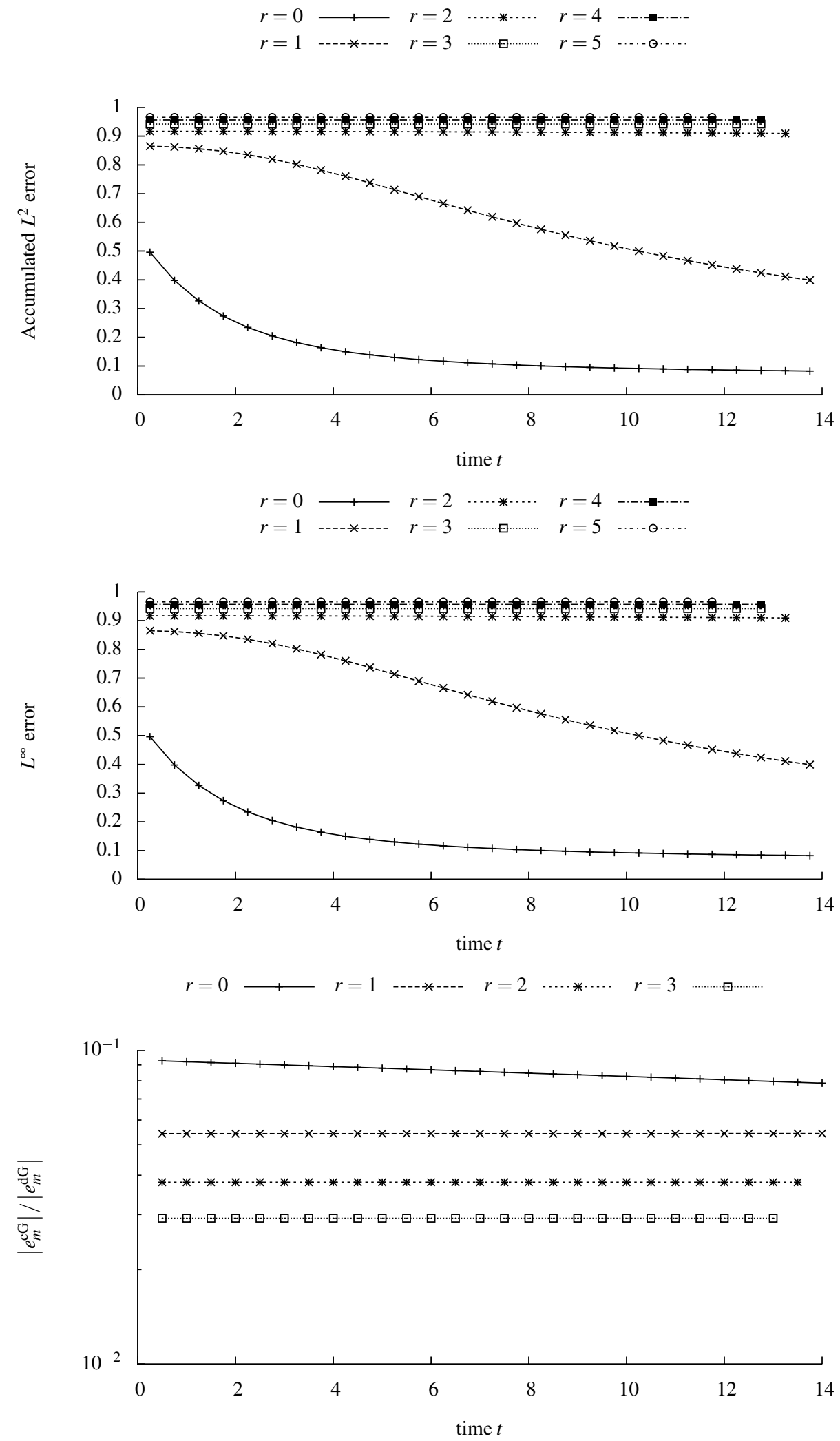

Fig. 1 Error ratios for $\beta=1$ (smooth solution) and $C_{\mathrm{ex}}=1$. 


$$
\begin{aligned}
& r=0 \quad \text { — } r=2 \quad \cdots \cdots * \cdots, \quad r=4 \quad \ldots-\cdots \\
& r=1 \quad---*---\quad r=3 \quad \cdots \cdots \cdots \cdots \cdots \cdots
\end{aligned}
$$

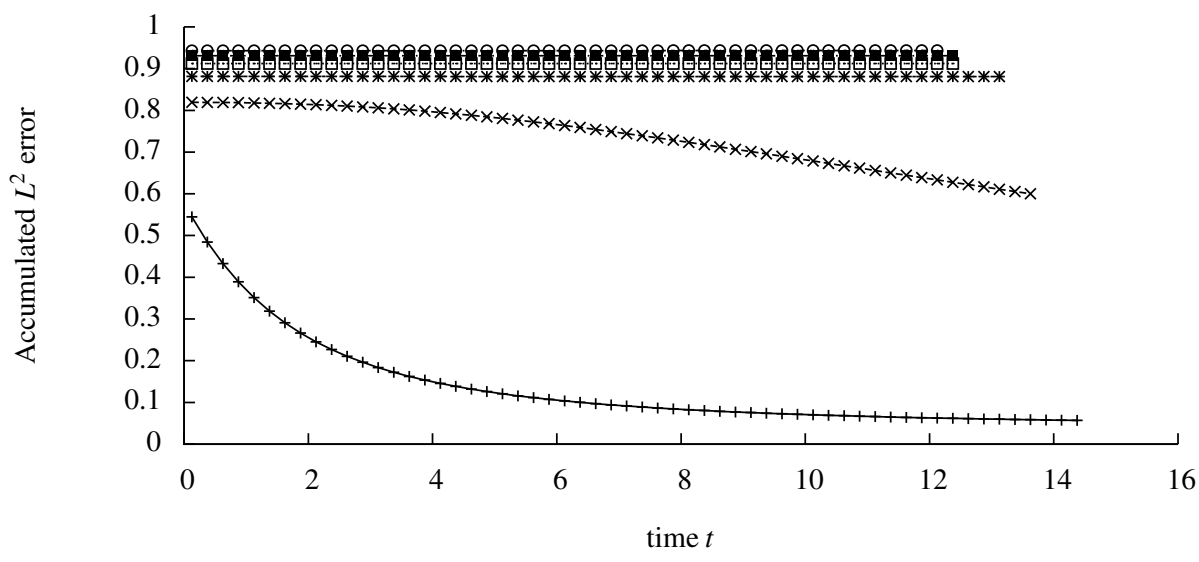

$$
\left.\begin{array}{llrllrl}
r=0 & 1 & r=2 & \cdots \cdots * \cdots \cdots & r=4 & \ldots
\end{array}\right]
$$
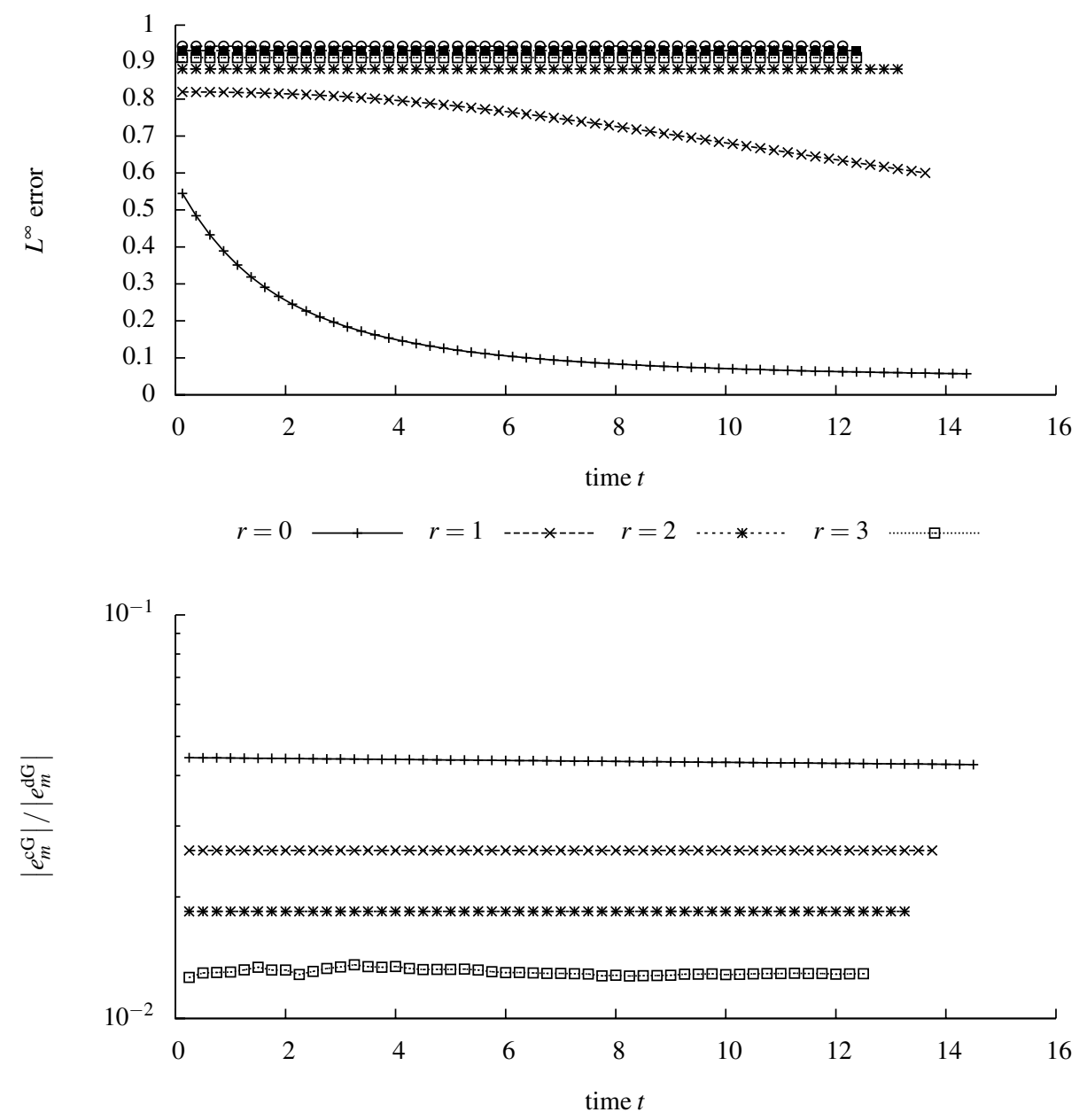

Fig. 2 Error ratios for $\beta=1$ (smooth solution) and $C_{\mathrm{ex}}=2$. 

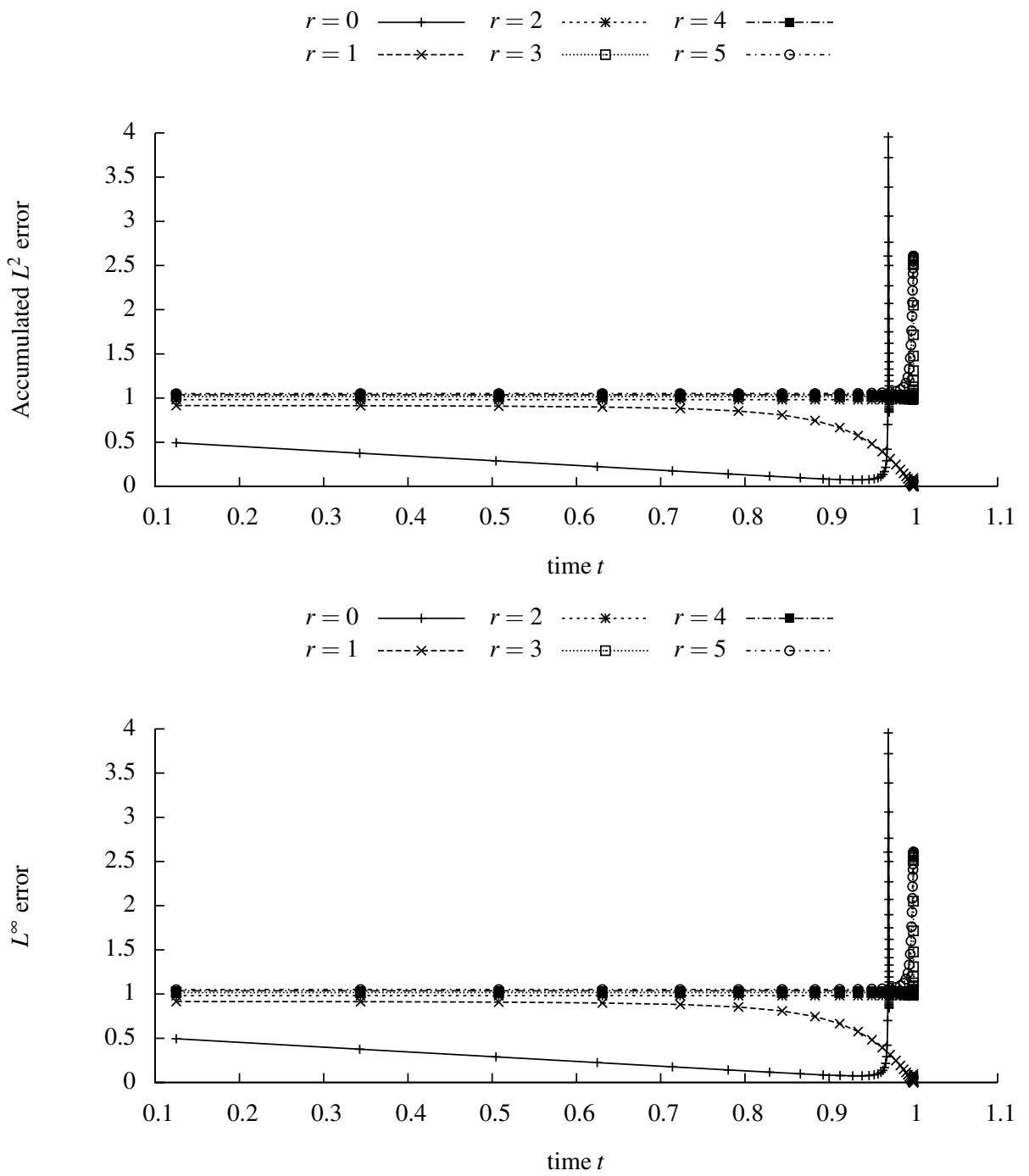

Fig. 3 Error ratios for $\beta=2$ (blow-up solution) and $C_{\mathrm{ex}}=1$.

3. Delfour, M., Hager, W., Trochu, F.: Discontinuous Galerkin methods for ordinary differential equations. Math. Comp. 36, 455-473 (1981)

4. Estep, D.: A posteriori error bounds, global error control for approximation of ordinary differential equations. SIAM J. Numer. Anal. 32, 1-48 (1995)

5. Estep, D., French, D.: Global error control for the continuous Galerkin finite element method for ordinary differential equations. RAIRO Modél. Math. Anal. Numér. 28, 815-852 (1994)

6. Janssen, B., Wihler, T.P.: Existence results for the continuous and discontinuous galerkin time stepping methods for nonlinear initial value problems. Submitted (2014)

7. Johnson, C.: Error estimates and adaptive time-step control for a class of one-step methods for stiff ordinary differential equations. SIAM J. Numer. Anal. 25, 908-926 (1988) 


$$
\begin{aligned}
& r=0 \quad r=2 \quad \cdots \cdots * \cdots \cdot r=4 \quad r \quad \cdots \\
& r=1 \quad \cdots \cdots *---\quad r=3 \quad \cdots \cdots \cdots \cdots \cdots \cdots \cdots
\end{aligned}
$$
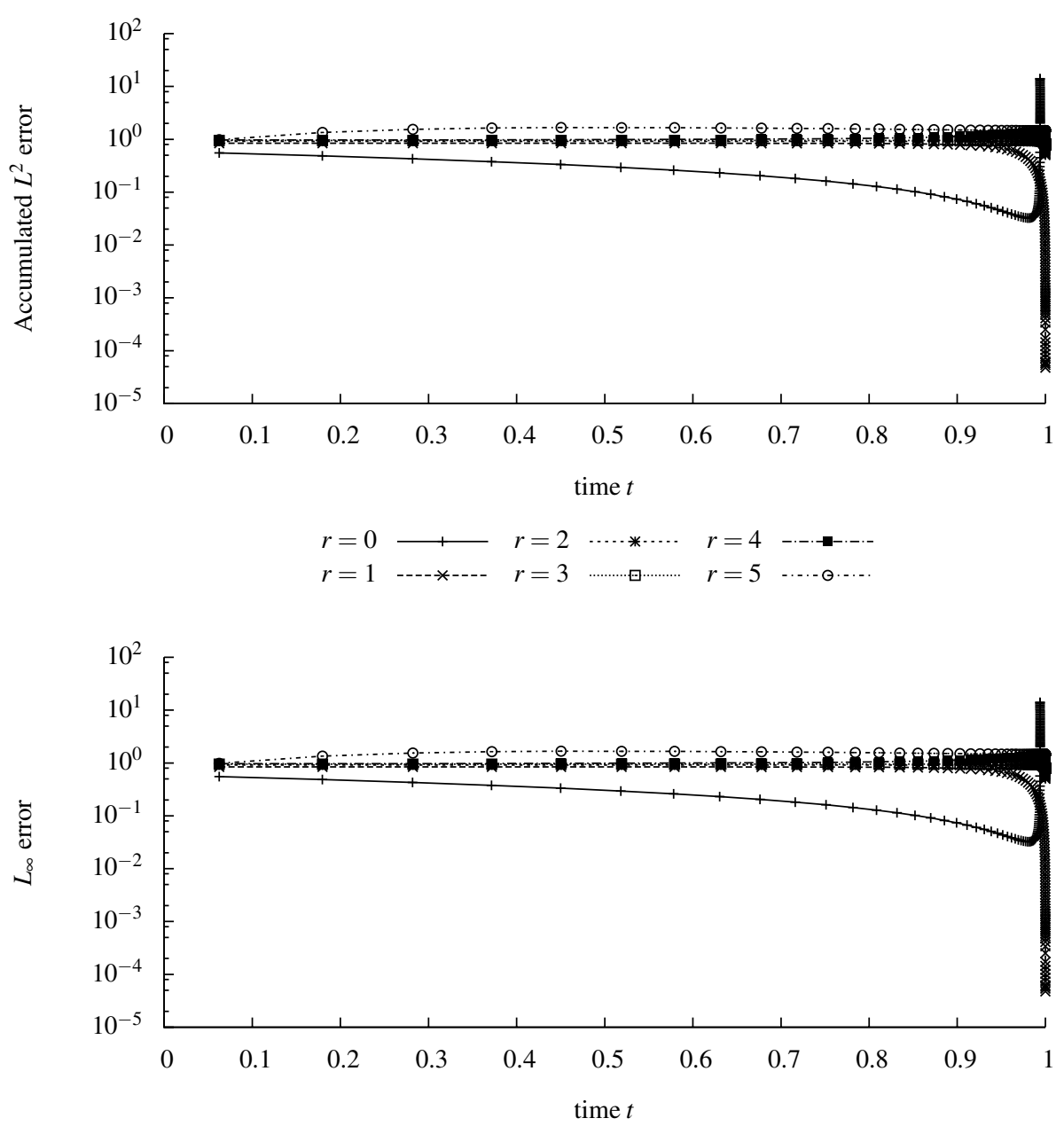

Fig. 4 Error ratios for $\beta=2$ (blow-up solution) and $C_{\mathrm{ex}}=2$.

8. Makridakis, C., Nochetto, R.H.: A posteriori error analysis for higher order dissipative methods for evolution problems. Numerische Mathematik 104(4), 489-514 (2006)

9. Mustapha, K., McLean, W.: Superconvergence of a discontinuous Galerkin method for fractional diffusion and wave equations. SIAM J. Numer. Anal. 51(1), 491-515 (2013)

10. Schötzau, D., Schwab, C.: An $h p$ a-priori error analysis of the DG time-stepping method for initial value problems. Calcolo 37, 207-232 (2000)

11. Schötzau, D., Wihler, T.P.: A posteriori error estimation for $h p$-version time-stepping methods for parabolic partial differential equations. Numer. Math. 115(3), 475-509 (2010). DOI 10.1007/s00211-009-0285-8. URL http://dx.doi.org/10.1007/s00211-009-0285-8 
12. Teschl, G.: Ordinary Differential Equations and Dynamical Systems, vol. 140, 9th edn. AMS (2012)

13. Wihler, T.P.: An a-priori error analysis of the $h p$-version of the continuous Galerkin FEM for nonlinear initial value problems. J. Sci. Comput. 25, 523-549 (2005) 